\title{
ADAPTIVE TEXTURE IMAGE RETRIEVAL IN TRANSFORM DOMAIN
}

\author{
Bin Zhang, Catalin I Tomai, and Aidong Zhang \\ Computer Science and Engineering Department, \\ State University of New York at Buffalo, NY 14226, USA. \\ Email: \{binzhang,citomai,azhang \}@cse.buffalo.edu
}

\begin{abstract}
A large number of algorithms have been proposed for texture image retrieval and analysis. While each algorithm has its own characteristics and is suitable for indexing some particular categories of images, there is a lacking of an effective strategy to integrate these algorithms to maximize the retrieval performance based on the algorithms available. Our first step in this direction is to build up a texture image retrieval system which adaptively chooses the "right" transform to query texture database. Experiments on the Brodatz texture set show that the adaptive image retrieval system significantly outperforms the commonly used single-algorithm based ones.

Index Terms- Content-based image retrieval, Texture analysis, Wavelets.
\end{abstract}

\section{INTRODUCTION}

Texture is an important property of many types of images. While there isn't a universally agreed definition of what a texture is ([7]), features of a texture include roughness, granulation and regularity.

Texture analysis is a challenging task. There is a large need to classify images based on textural features in various fields like scene analysis, medical images analysis, etc. Various texture analysis systems were proposed over the years (see reviews in [4], [5], [7]). Three different types of texture analysis are considered: segmentation, classification and synthesis. Texture segmentation deals with detecting the texture boundaries in an image to obtain a boundary map. Texture classification deals with the recognition of image regions using texture properties. Each region in an image is assigned a texture class. The goal of texture synthesis is to extract three-dimensional information from texture properties.

Randen et al. ([4]) have compared different approaches to texture characterization using filtering and statistical methods. Filtering approaches include: Laws masks, ring/wedge filters, discrete cosine transforms, eigenfilters, optimized Gabor filters, linear predictors, dyadic Gabor filter-banks, etc. Statistical approaches were among the first proposed in the literature and include co-occurrence, and autoregression methods. The conclusion of the survey was that various filtering techniques produce different results for different images and therefore no single approach performs best for all images. However, algorithms using wavelet transforms achieve consistently good performance and rank among the best approaches.

For wavelet-based multiresolution decomposition, simple features like means, variances, and moments are ex- tracted from the wavelet subbands at each decomposition level. Among various wavelets, Gabor filter banks have been found to have the best performance in texture image retrieval ([2]). The Gabor filter banks consist of arbitrarily scaled and rotated filters ([6]) which extract texture characteristics (locality/resolution) in both spatial and spatialfrequency domains.

As stated in [3], humans tend to use different types of information rather than just one classification method when recognizing similar patterns. Hence, we may expect that a system that combines several texture classification methods in an efficient way would be closer to the human performance. Our first step in this direction is a supervised texture-classification scheme that adaptively chooses the "best" transform for a certain texture.

Inspired by the observations above, we designed an Adaptive Content-Based Image Retrieval System (ACBIRS) where different wavelet filters are dynamically selected to adapt different query images to achieve an "optimal" performance with regard to the given wavelet set.

The rest of the paper is organized as follows. Section 2 presents the A-CBIRS architecture. Section 3 describes the texture feature extraction process based on the transforms. Section 4 describes the training process and Section 5 details the image retrieval process. Experimental results are presented and analyzed in Section 6. Section 7 contains conclusions and remarks.

\section{THE SYSTEM ARCHITECTURE OF A-CBIRS}

We first specify the problem of A-CBIRS, then present our A-CBIRS architecture based on k-nearest neighbor search.

\subsection{Problem Description}

Let $C=\left\{c_{1}, c_{2}, \ldots, c_{K}\right\}$ be a set of $\mathrm{K}$ texture classes, and $\mathcal{F}=\left\{f_{1}, f_{2}, \ldots, f_{\aleph}\right\}$ be a set of $\aleph$ CBIR algorithms. For each texture class $c \in C$, the $\aleph$ algorithms are ranked by their retrieval performance $r$ like recall or precision, i.e., $\mathcal{F}^{c}=\left\{\left(f_{i}^{c}, r_{i}^{c}\right) \mid r_{i}^{c} \leq r_{j}^{c}, \forall 1 \leq j<i \leq \aleph\right\}$.

Let $\mathcal{S}$ be a set of images to be indexed. The objective of A-CBIRS is to maximize the overall retrieval performance on $\mathcal{S}$ by selecting a particular CBIR algorithm $f_{I}$ in $\mathcal{F}$ for each image $I(\in \mathcal{S})$ belonging to $c$ class such that $r_{f_{I}}$ approaches $f_{1}^{c}$.

\subsection{The System Architecture of A-CBIRS}

To solve the above problem we set up a training image set $\mathcal{L}$ which contains as many texture classes as possible. Then, 
in the training process we build the $\mathcal{F}^{c}$ mapping set. We use an approximation algorithm to compute the features for the query image $I$ and the images in $\mathcal{L}$. Then, we apply the k-nearest neighbor search over $\mathcal{L}$ and determine the set of images "closest" to $I$. The search can be performed over the entire set of feature vectors obtained for the images of $\mathcal{L}$ or a reduced set of representative feature vectors.

Therefore, there are two main stages to design ACBIRS: the training process and the query process, shown as Figure 1. During the training process, for each texture category, the algorithms in $\mathcal{F}$ are ranked by average retrieval performance on the training image database (TIDb). The ranking information is stored in a category-algorithm database $\Gamma$ indexed by texture category. The training process produces a training feature database TFDb, which contains feature vectors obtained by applying each algorithm in $\mathcal{F}$ on each image in TIDb. From $\mathcal{F}$, a mean feature database $M F D b$ is also generated by computing the mean of all feature vectors belonging to the identical category. $\mathcal{F}$ has $\mathrm{K}$ entries corresponding to $\mathrm{K}$ texture classes considered.

Given a query icon $\chi$, the query process first applies an approximate algorithm on it, then queries TFDb, and labels $\chi$ with a category $\varsigma$. The category label is determined by majority voting considering the top ten entries retrieved. With $\varsigma, \Gamma$ is indexed to get the optimal(best) wavelet transform $B_{a g}$. Finally, based on $B_{a g}$ transform features, we query the testing (query) image database (QIDb) with the given icon $\chi$.

\section{TRANSFORM FEATURE EXTRACTION}

To simplify the system, we take into account $16(\aleph=$ 16) transforms as the CBIRS algorithm pool of $\mathcal{F}$ : DCT (w0), Haar (w1), Daubechies 4 (w2), Daubechies 6 (w3), Daubechies 8 (w4), Antonini (w5), Villa (w6), Adelson (w7), Brislawn (w8), Brislawn 2 (w9), Villa 2 (w10), Villa 4 (w11), Villa 6 (w12), Odegard (w13), Dyadic Mallat (w14), and Gabor filter bank (w15). Features are extracted in transform domain. A Gabor filter bank and a measure based on Grey Level Coocurrence Matrices (GLCM) would be the approximation algorithms.

\subsection{Feature Extraction}

The DCT transform is performed once on an image, and the 9 coefficients from the uppermost left $3 \times 3$ matrix are taken as features, representing the energy in the lower frequencies.

For an image $I$, we perform a particular wavelet transform twice. The first transform is applied on $I$ itself, resulting in four subbands: LL, LH, HL, HH. The second transform is performed on the subband LL, resulting in another 4 subbands, LLLL, LLLH, LLHL, LLHH. Therefore, the total number of subbands from the two transformations are 7. For each of the seven subbands, we compute their mean absolute value and variance as it's features. In total, a 14-dimensional feature vector is obtained for $I$.
In A-CBIRS, the Gabor filter bank consists of 30 Gabor filters covering 5 scales and six orientations. We apply such a bank of Gabor filters on $I$ and obtain 30 filtered images. For each of the 30 filtered images we calculate it's mean absolute value and variance as it's features. Thereby, a total of 60 Gabor transform features are produced.

The GLCM algorithm [1] calculates textural features based on spatial dependence matrices at 0, 45, 90 and 135 degrees for four distances as 1,2,3, and 4. There are a total of 52 GLCM features.

For simplification, we use a feature vector $V$ of $\mathrm{N}$ dimensions to represent any of the four types of feature vectors, the 9-dimensional DCT transform one, 14dimensional wavelet transform one, the 60-dimensional Gabor transform one, and 52-dimensional GLCM one.

$$
V=\left(v_{1}, v_{2}, \cdots, v_{N}\right)
$$

A feature vector $V$ is associated with a specific transform from $\aleph$ transforms. Therefore, for any image $I$, there are $\aleph$ feature vectors corresponding to $\aleph$ transforms.

Let $\Omega$ be the $\mathrm{N}$-dimensional vector space and $\psi$ represent $\aleph$ feature vectors of an image, then

$$
\psi=\left\{\tau, V^{1}, V^{2}, \ldots, V^{\aleph} \mid V^{i} \in \Omega, \forall 1 \leq i \leq \aleph\right\}
$$

where $\tau$ is the truth class of the texture image $I$.

\subsection{Distance Measures}

Given two feature vectors $X \in \Omega$ and $Y \in \Omega$ from the same transform, two distance measures are used in the system. For querying QFDb or TFDb based on transform features, the Manhattan distance is used:

$$
d(X, Y)=\sum_{i=1}^{N}\left|x_{i}-y_{i}\right|
$$

When querying TFDb or MFDb based on GLCM features, we use a variation of Manhattan distance as:

$$
d(X, Y)=\sum_{i=1}^{N} \frac{\left|x_{i}-y_{i}\right|}{\alpha_{i}}
$$

where $\alpha_{i}$ is the standard deviation of the respective feature over the entire training database $T F D b$.

In the distance measure (4), each feature component is normalized by its corresponding standard deviation so that all feature components can be compared in the same scale. Values of different GLCM features span much different ranges, e.g., sum variance may amount to $1.045 e+04$ while angular second moment is as small as $4.042 e-04$. Normalization avoids the dominant effect of some component features.

\subsection{Feature Database}

The build-up of the feature database (FDb) is done by computing all the feature vectors for each image in an image database (IDb) and storing these vectors in one or more formatted file (database). Querying FDb instead of IDb itself will speed up the query process. 


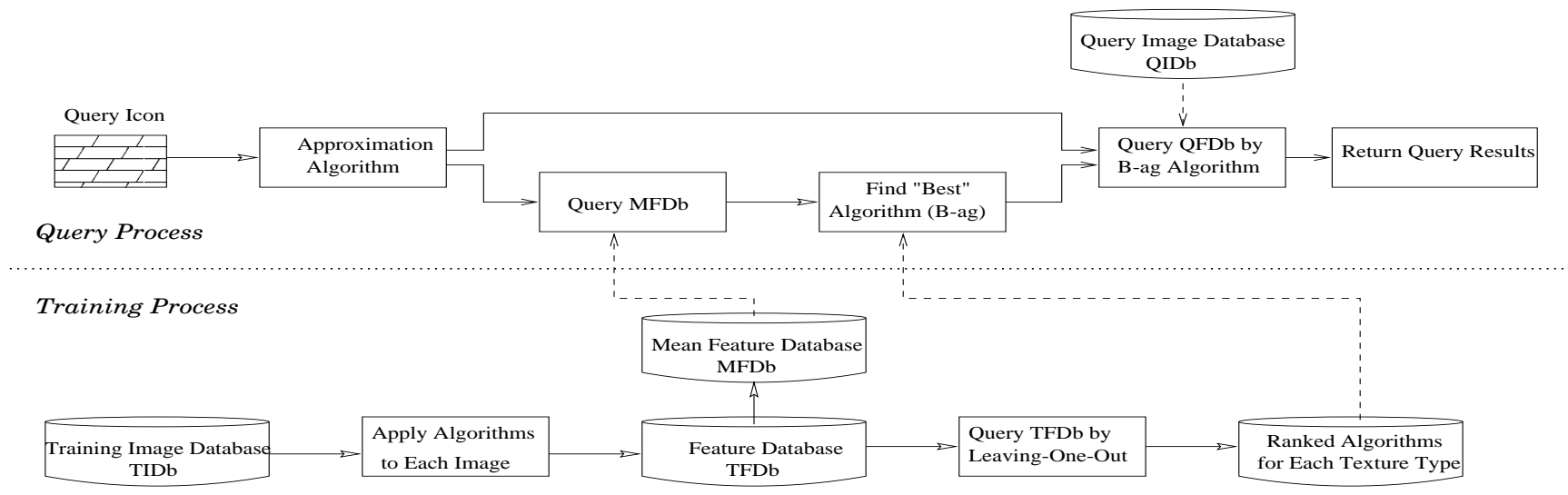

Fig. 1. The System Architecture of A-CBIRS

Given an image database IDb with $\mathrm{M}$ images, it's FDb can be described as:

$$
F D b=\left\{\psi_{1}, \psi_{2}, \ldots, \psi_{M}\right\}
$$

The mean feature database MFDb contains the mean feature vectors for each texture category over some image set. MFDb is structured as:

$$
M F D b=\left\{\psi_{i} \mid \psi_{i} \cdot \tau=c_{i}, 1 \leq i \leq K\right\},
$$

where $\mathrm{K}$ is the total number of texture classes.

\section{TRAINING PROCESS}

The lower diagram in Figure 1 displays the training process.

Given a training image database (TIDb), the training process first builds up the corresponding feature database $\mathrm{TFDb}$ as discussed in the previous section. Then, we query TFDb with every feature vector in TFDb based on the distance measure (3) with self matches excluded.

Let $X=T F D b . \psi_{i} . V^{j}$, indicating the $i$ th image's features based on the $j$ th transform, then, all the distances computed form a set as,

$D_{i}^{j}=\left\{d_{i k}=d\left(X, T F D b . \psi_{k} . V^{j}\right) \mid 1 \leq k \leq M\right.$ and $\left.k \neq i\right\}$

The distances in $D_{i}^{j}$ are sorted in ascending order. The retrieval effectiveness is measured by recall of $\gamma$ defined as (8). The recall for querying $F D b$ with $X$ is $\gamma_{i}^{j}$.

$$
\gamma=\frac{\text { number }- \text { of }- \text { relevant }- \text { retrieved }}{\text { number }- \text { of }- \text { relevant }}
$$

The process above is repeated for all $i$ and $j, \forall 1 \leq$ $i \leq M, \forall 1 \leq j \leq \aleph$. Then, with regard to each transform, we can compute the average recall from the recall set $\left\{\gamma_{i}^{j} \mid \forall 1 \leq i \leq M, \forall 1 \leq j \leq \aleph\right\}$ for each texture category. Finally, for each texture category, all transforms are ranked by their average recalls.

For each texture category, a transform $w$ is associated with a recall $\gamma$, a structure like $T=\{w, \gamma\}$ is used to describe this set-up. Then the training result of $\Gamma$ on TIDb with $\Im$ texture categories can be stated as (9).

$$
\Gamma=\left\{T_{i} \mid 1 \leq i \leq \Im\right\}
$$

After the training process, we obtain three databases, the training feature database TFDb, the mean feature database for $\mathrm{K}$ texture classes, and the training result database $\Gamma$. In the next section, we'll show how to use this information for query processing.

\section{QUERY PROCESS}

The upper diagram in Figure 1 displays the query process.

Given a query icon $\hbar$, we first compute its feature vector $X$ by applying the approximation algorithm, then query either TFDb or MFDb with $X$ in the following ways:

$$
\begin{aligned}
& D^{g}=\left\{d_{k}=d\left(X, T F D b . \psi_{k} . V^{g}\right) \mid 1 \leq k \leq M\right\} \\
& D^{g}=\left\{d_{k}=d\left(X, M F D b . \psi_{k} . V^{g}\right) \mid 1 \leq k \leq K\right\}
\end{aligned}
$$

where $g$ is the index of the approximation algorithm.

The top $k$ ( $k=10$ in A-CBIRS) closest entries will be chosen as,

$$
Q^{g}=\left\{\psi_{1}^{g}, \psi_{2}^{g}, \ldots, \psi_{k}^{g}\right\}
$$

From $Q^{g}$, a weighted (by recall) majority voting method is used to choose the "optimal" algorithm $B_{a g}$.

Eventually, based on the $B_{a g}$ transform, we perform retrieval over the query image database QIDb with the query icon $\hbar$. If $B_{a g}$ is equal to $g$, $\hbar$ 's feature vector does not need to be calculated again.

\section{EXPERIMENTAL RESULTS AND ANALYSIS}

\subsection{Brodatz Texture Database}

We have used the Brodatz set of textures as our working set. While the Brodatz set represents only a subset of a larger set of textures of interest it is still useful for performance evaluation.

Each Brodatz texture image is digitized into a $512 \times 512$, 256 grey-level image and cut into 20 subimages of size $96 \times$ 96. The whole set of 20 subimages compose the relevant set for that particular texture. We have randomly divided 
this set of 20 images into a training set (10 subimages) and a testing set (10 subimages) and therefore we have a total of $1120=112 \times 10$ images in the training set of TIDb and 1120 in the query set of QIDb.

\subsection{Training Results}

Given $\aleph(\aleph=16)$ transforms and the training image database TIDb, we follow the steps in Section 4 to build up a feature database TFDb and a training result database $\Gamma$.

Table 1 presents the performance ranking of $\aleph$ transforms for some images of the Brodatz set.

From the statistics about the frequency of a certain transform performs best on the entire set of textures, we found that the Gabor transform outperforms the other transforms in almost half the cases. DCT transform performs the worse among the entire set of filters. There are cases in which several transforms share the same rank, that is they present the same performance on the training set.

\subsection{Query Results}

We have set up four different schemes to test the performance of adaptive image retrieval depending on how to compute the optimal algorithm for a query icon:

1. Scheme 1: use Gabor filter bank to query TFDb.

2. Scheme 2: use Gabor filter bank to query MFDb.

3. Scheme 3: use GLCM to query TFDb.

4. Scheme 4: use GLCM to query MFDb.

Figure 2 presents the performances for 16 transforms with the Gabor filter bank outperforming the other transforms slightly. Figure 3 shows the performance comparison of the proposed four adaptive schemes and the Gabor filter bank. In both figures, the $x$ axis represents the number of top images retrieved and the $y$ axis shows the corresponding average retrieval rate computed on the testing set.

Figure 4 shows how many times each transform was used in the four schemes. The Gabor filter bank was used most frequently.

For Scheme 1, the number of texture images that show a classification rate in a certain percentage interval are presented in Table 2. The fourth column shows the number of times the average retrieval rate belongs to a certain interval when always using the "best" algorithms for the testing set. The third column shows the results from Scheme 1 where the pre-determined Gabor filter bank is used for choosing the optimal algorithms. The second column shows the performance of the Gabor transform on the testing set. Even if the adaptive system using Scheme 1 does not reach the "ideal" performance it still presents a clear improvement over using only the Gabor transform retrieval.

Table 4 presents some examples of images retrieved from the testing set when using Scheme 1. Table 5 presents the textures retrieved for the same query icons when using the transform chosen by the adaptive system. The first

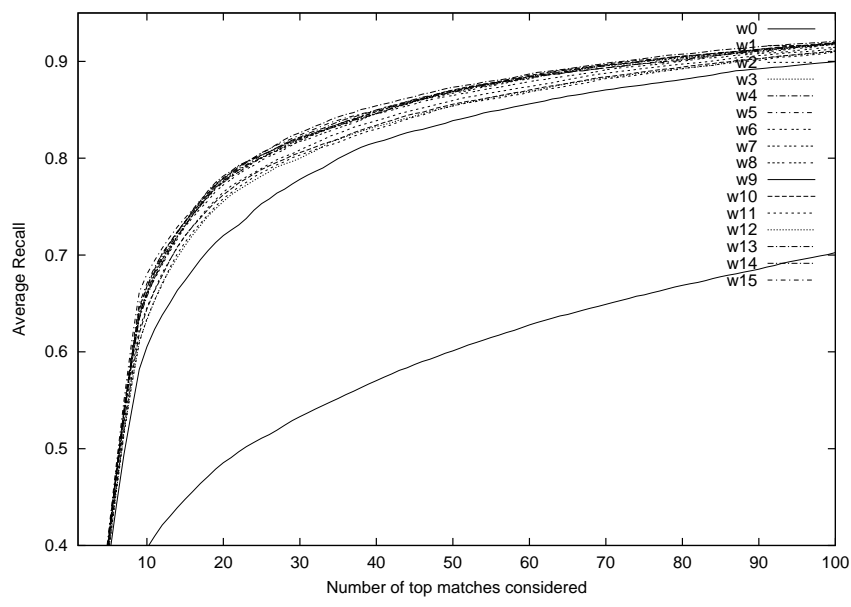

Fig. 2. Comparison of retrieval performance of different features based on 16 different transforms.

\begin{tabular}{|c|c|c|c|}
\hline Retrieval Rate & Gabor & Adaptive & Best \\
\hline $100 \%-90 \%$ & 48 & 53 & 58 \\
\hline $89 \%-80 \%$ & 10 & 13 & 13 \\
\hline $79 \%-70 \%$ & 10 & 9 & 10 \\
\hline $69 \%-60 \%$ & 13 & 7 & 8 \\
\hline $59 \%-0 \%$ & 31 & 30 & 23 \\
\hline
\end{tabular}

Table 2. Classification results for the 112 Brodatz textures in Scheme 1.

column indicates the transform index, the other columns contain the image name and the actual image for the query texture and the top 5 textures retrieved.

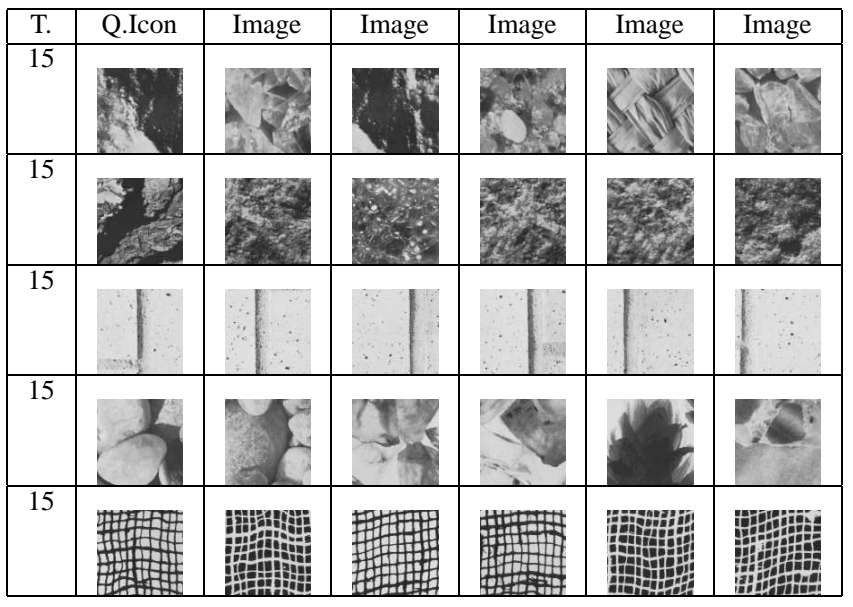

Table 3. Textures retrieved using the Gabor transform for some random query images

\subsection{Analysis}

According to Figure 2, the Gabor filter bank performs better than the other transforms while DCT presents the worst performance, which confirm the conclusions in [2]. As shown in Figure 3, all four adaptive schemes consistently outperform the Gabor filter bank. Given any number $(L)$ of 


\begin{tabular}{|c|c|c|c|c|c|c|c|c|c|c|c|c|c|c|c|c|}
\hline Image & w0 & w1 & w2 & w3 & w4 & w5 & w6 & w7 & w8 & w9 & w10 & w11 & w12 & w13 & w14 & w15 \\
\hline d002 & 15 & 13 & 10 & 7 & 14 & 8 & 5 & 1 & 6 & 9 & 12 & 11 & 2 & 4 & 3 & 0 \\
\hline $\mathrm{d} 010$ & 1 & 2 & 3 & 4 & 15 & 5 & 6 & 8 & 10 & 13 & 7 & 11 & 12 & 9 & 14 & 0 \\
\hline $\mathrm{d} 043$ & 15 & 0 & 9 & 1 & 13 & 14 & 10 & 7 & 8 & 11 & 12 & 2 & 5 & 6 & 3 & 4 \\
\hline $\mathrm{d} 044$ & 0 & 15 & 4 & 1 & 6 & 14 & 3 & 2 & 8 & 9 & 10 & 11 & 12 & 13 & 5 & 7 \\
\hline $\mathrm{d} 112$ & 10 & 13 & 6 & 8 & 11 & 5 & 7 & 12 & 2 & 3 & 4 & 9 & 15 & 14 & 1 & 0 \\
\hline
\end{tabular}

Table 1. Performances of different wavelets on different textures

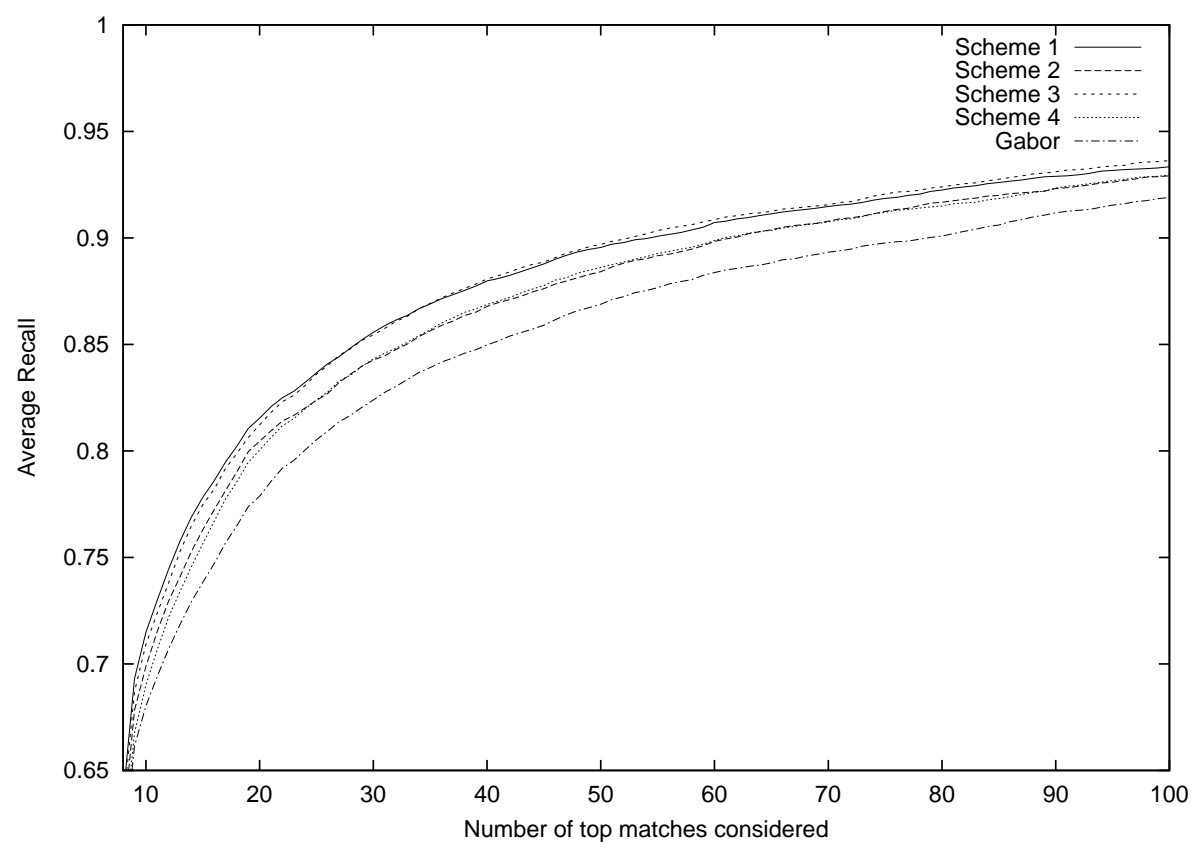

Fig. 3. Performance comparison of the four adaptive schemes and the Gabor transform (w15).

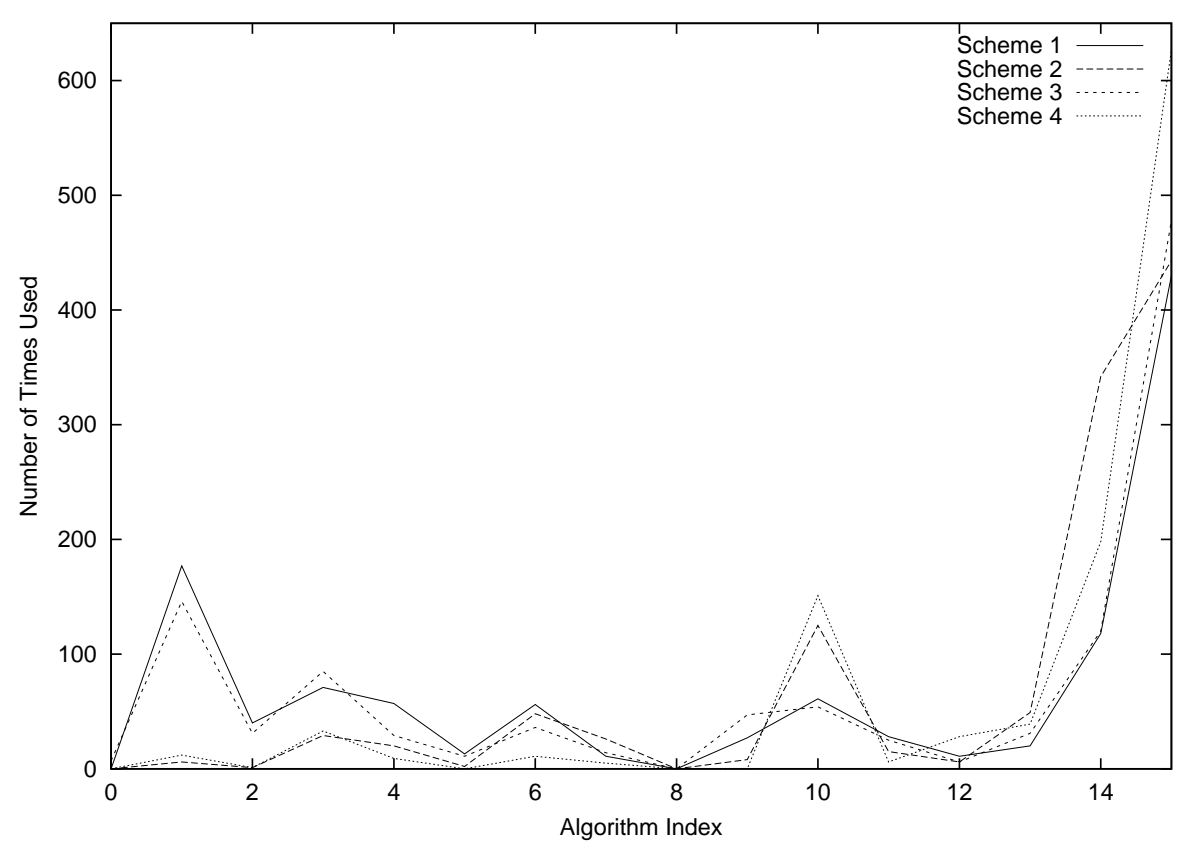

Fig. 4. Number of times for each transform used in the four schemes. 


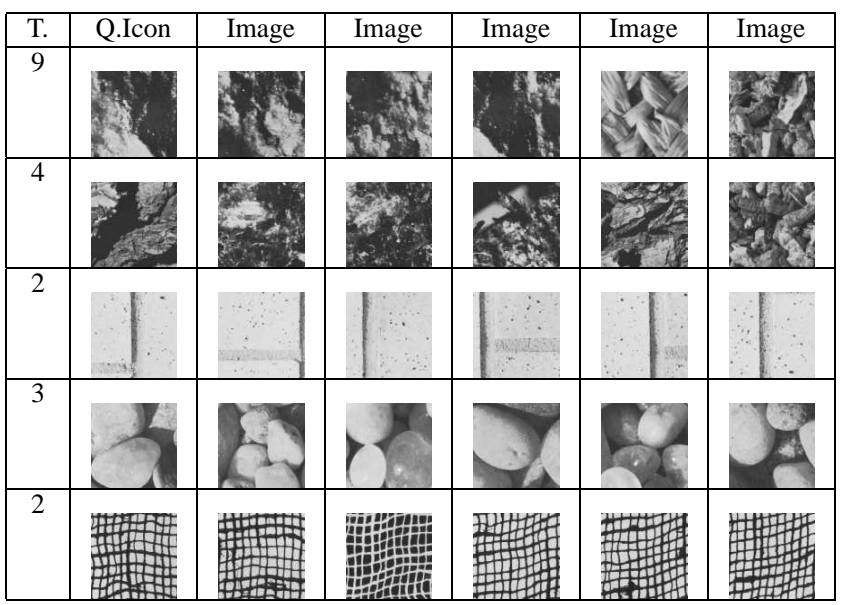

Table 4. Textures retrieved using the transform chosen by A-CBIRS for some random query images

top images retrieved, the average recalls of all four schemes are always higher than that of the Gabor filter bank. For $L=9$, the average recall using Scheme 1 is $69.13 \%$, about $3 \%$ higher than that of the Gabor filter bank, a very significant improvement. With $L=9$, the average recall using Scheme 2 gets to $67.83 \%$, the one using Scheme 3 amounts to $68.70 \%$, and the one using Scheme 4 is $66.77 \%$. When $L \leq 25$, the two adaptive schemes (Scheme 1 and Scheme 2) using the Gabor filter bank gives higher retrieval accuracy than that of the two using GLCM (Scheme 3 and Scheme 4) respectively; When $L>25$, GLCM slightly outperforms the Gabor filter bank.

From the experiments, we observe that while in some cases the performance of the transform chosen by the adaptive system is slightly worse than the Gabor transform performance ( e.g. images d005, d023, d064) the performance is dramatically higher for other images (e.g. images d028, d074, d075, etc). Further investigation is necessary to determine the causes of this variance in the performance.

On the other hand, compared with the best transform, the overall performance of Scheme 1 is about $4.6 \%$ lower. This difference means that there is still much room for improving the transform selection method. One way to correct the bias caused by using the Gabor filter to coarsely classify a query icon prior to querying QIDb is to choose a different method from Gabor. GLCM is an option for correcting the Gabor bias.

As the size of MFDb is only $10 \%$ of that of QFDb, choosing the optimal algorithm through querying MFDb increases computation by only $10 \%$. Computation overhead is minimized by querying MFDb instead of TFDb.

Obviously pre-classification by querying MFDb (based on both Gabor and GLCM features) gives lower accuracy because computing the mean values reduces the representativeness of the set. There is a tradeoff between the retrieval performance and the computation cost. It is expected that a medium-size TFDb with reasonable diversity of textures will boost the retrieval accuracy of the adaptive image retrieval system with small computation overhead.

\section{CONCLUSIONS AND FUTURE WORK}

In this paper, we have proposed four adaptive schemes, which significantly outperform a retrieval system that would use any single algorithm. The overhead for computing the optimal transform in adaptive retrieval is dramatically reduced by precomputing the features for the textures in the training database and generating a corresponding mean feature database. Future work would concentrate on using other features besides the Gabor and GLCM features to preclassifiy a query icon. Moreover, more algorithms will be added to the A-CBIRS setup, potentially increasing its current performance.

\section{REFERENCES}

[1] R. M. Haralick, K. Shanmuga, and I. Dinstein. Textural features for image classification. IEEE Transactions on Systems, Man, and Cybertinetics, SMC-3(6):610-621, 1973.

[2] W. Ma and B. Manjunath. Texture features for browsing and retrieval of image data. Pattern Analysis and Machine Intelligence, pages 837-842, 1996.

[3] J. S. Payne and T. Stonham. Can texture and image content retrieval methods match human perception? In Proc. International Symposium on Intelligent Multimedia, Video and Speech Processing, 2001.

[4] T. Randen and J. H. Husoy. Filtering for texture classification: A comparative study. IEEE Transactions on Pattern Analysis and Machine Intelligence, 21(4):291310, 1999.

[5] T. Reed and J. Buf. A review of recent texture segmentation and feature extraction techniques. CVGIP: Image Understanding, 57(3):359-372, 1993.

[6] J. R. Smith and S.-F. Chang. Quad-tree segmentation for texture-based image query. In Proceedings of the 2nd Annual ACM Multimedia Conference, San Francisco Ca, October 1994.

[7] M. Tuceryan and A. Jain. Texture analysis. In In C. H Chen, L. F. Pau, and P.S. P. Wang, editors, The Handbook of Pattern Recogntion and Computer Vision, pages 207-248. World Scientic Publishing Co., 1998. 